Documentation et bibliothèques

DOCUMENTATION BIBLIOTHËUES

\title{
Le catalogue réinventé
}

The Library Catalogue Renewed

El catálogo reinventado

\section{Charles-Antoine Julien et France Bouthillier}

Volume 54, numéro 3, juillet-septembre 2008

URI : https://id.erudit.org/iderudit/1029200ar

DOI : https://doi.org/10.7202/1029200ar

Aller au sommaire du numéro

\section{Éditeur(s)}

Association pour l'avancement des sciences et des techniques de la documentation (ASTED)

ISSN

0315-2340 (imprimé)

2291-8949 (numérique)

Découvrir la revue

Citer cet article

Julien, C.-A. \& Bouthillier, F. (2008). Le catalogue réinventé. Documentation et bibliothèques, 54(3), 229-239. https://doi.org/10.7202/1029200ar

\section{Résumé de l'article}

Le catalogue en tant qu'outil de recherche offert au public fut conçu pour faciliter le repérage de ressources documentaires spécifiques à l'aide d'informations précises, telles que le titre et le nom de l'auteur. Or, le catalogue est de moins en moins pertinent aux yeux des chercheurs d'aujourd'hui ayant accès à de multiples outils en ligne plus conviviaux et dynamiques. Quelques bibliothèques tentent de regagner la faveur des chercheurs en réinventant le catalogue traditionnel pour qu'il soit plus visuel, interactif et convivial. Cet article décrit une nouvelle génération de catalogues de bibliothèques accessibles au public afin d'aider les institutions qui voudraient s'en inspirer. Il reprend, en l'enrichissant, le contenu d'une présentation faite au récent Colloque conjoint CBPQ/ASTED, tenu en mai 2008 à Montréal.
Tous droits réservés (C) Association pour l'avancement des sciences et des techniques de la documentation (ASTED), 2008
Ce document est protégé par la loi sur le droit d'auteur. L'utilisation des services d’Érudit (y compris la reproduction) est assujettie à sa politique d'utilisation que vous pouvez consulter en ligne.

https://apropos.erudit.org/fr/usagers/politique-dutilisation/ 


\section{Le catalogue réinventé}

Charles-Antoine Julien

Doctorant

charles.julien@mcgill.ca

\section{FRANCE BOUTHILLIER}

Professeure agrégée et directrice france.bouthillier@mcgill.ca

École des sciences de l'information Université McGill

Montréal, Qc.

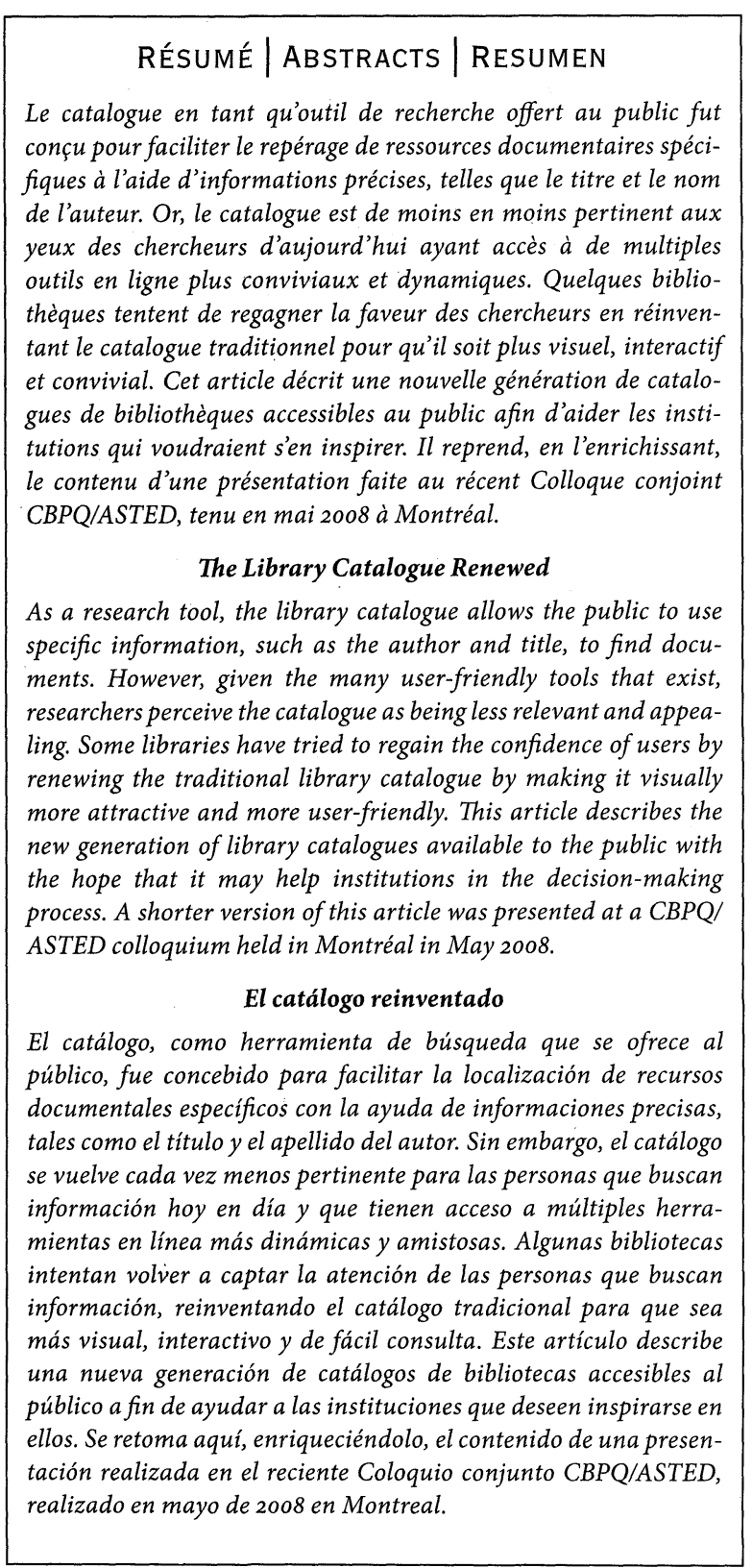

\section{Introduction}

De nombreuses publications ${ }^{1}$ suggèrent que le catalogue de bibliothèque traditionnel ne répond plus aux attentes des chercheurs et que les statistiques d'utilisation diminuent constamment (Calhoun, 2006). Plusieurs bibliothèques à travers le monde se demandent comment faire évoluer leur catalogue public et ainsi redorer leur image en ligne compte tenu de la concurrence grandissante et de l'innovation constante. Les bibliothèques québécoises font également face à cette situation si on en juge par la décision du Sous-comité des bibliothèques de la $\mathrm{CREPUQ}^{2}$ de confier à son Groupe de travail sur le traitement de la documentation (GTTD) le mandat d'" établir un état de la réflexion actuellement en cours quant aux catalogues de bibliothèques et à l'accès aux collections dans un contexte compétitif d'accès à l'information ${ }^{3}$; le rapport du GTTD sera soumis au Sous-comité des bibliothèques durant l'automne 2008:

Notre article vient nourrir cette réflexion concernant l'avenir du catalogue en tant qu'outil de recherche d'information et son rôle dans la construction de l'image virtuelle de la bibliothèque. Nous débuterons en décrivant les attentes des chercheurs d'aujourd'hui, attentes fortement influencées par les initiatives d'entreprises issues du Web (par exemple Google et Amazon). Cet état de la question sera suivi d'un examen de l'état actuel du catalogue traditionnel et de ses faiblesses. Par la suite, nous présenterons trois catalogues novateurs, représentatifs de cette nouvelle génération de catalogues amorcée en 2006 par l'installation d'Endeca à l'Université de la Caroline du Nord, d'Aquabrowser dans plus de 250 bibliothèques à travers le monde et du catalogue hébergé Worldcat Local implanté à l'Université de Washington par l'entreprise OCLC (Online Computer Library Center).

Les grands éditeurs de systèmes intégrés de gestion de bibliothèque (SIGB) ont réagi face à ce changement 
Le chercheur d'aujourd'hui a droit à un large éventail d'outils de recherche et, s'il doit apprendre à les utiliser, il optera pour ceux qui le dispenseront d'apprentissage.

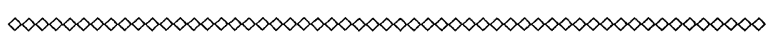

de fond en proposant de nouvelles versions des modules de gestion des catalogues. Par ailleurs, le dynamique domaine du logiciel libre offre également des solutions éprouvées pour les petits et grands réseaux de bibliothèques. Après une description de ces nouvelles tendances, l'article offre, en conclusion, quelques recommandations pour les institutions qui tentent d'établir la marche à suivre afin de faire évoluer le catalogue qu'elles offrent à leurs usagers.

Pour les fins de cet article, le terme " catalogue " réfère exclusivement à l'outil de recherche offert au public et n'inclut pas les fonctions reliées aux processus internes de la bibliothèque (achats, catalogage, prêt, etc.). Le terme " catalogue traditionnel » décrit le processus de recherche privilégié par la majorité des catalogues actuels en ligne soit :

- le choix de l'index;

- l'entrée de mots-clés;

- la présentation d'une liste de résultats triés par date de publication.

De plus, dans cet article, la notion de catalogue se limite exclusivement à l'outil d'accès à l'information offert par la bibliothèque aux utilisateurs, sans égard à la qualité de cette information. Nous évitons donc le débat concernant le processus de production des notices bibliographiques (le catalogage en tant que tel), leur qualité, leur utilité ou leur origine. Bien que ces sujets soient fortement reliés, nous croyons que le catalogue traditionnel est de moins en moins utilisé en raison de barrières purement technologiques. En effet, peu importe la qualité de l'information, un chercheur ne la trouvera pas s'il n'est pas intéressé à utiliser le catalogue. Une fois cette tendance inversée, il sera plus facile de justifier une bonification du contenu accessible au moyen d'un outil à nouveau apprécié par le public.

\section{La situation actuelle du catalogue en ligne}

Une série de facteurs dresse un portrait sombre de l'état actuel du catalogue en commençant par la fin de son monopole, avec l'arrivée du Web vers 1995 (Markey, 2007) comme outil de recherche d'information accessible au grand public. Depuis, le marché de la recherche d'information en ligne évolue très rapidement en offrant des outils performants, attrayants et appréciés par une population d'internautes à croissance exponentielle. Aujourd'hui, la grande majorité (Calhoun, 2006) des recherches d'information en ligne sont effectuées à l'aide d'un moteur de recherche Web tel que Google ou Yahoo !, et certains ${ }^{4}$ ont suggéré qu'il est plus facile et plus agréable de trouver un livre chez un libraire en ligne comme Amazon que dans le catalogue de la bibliothèque.

Cette situation s'explique par certains faits historiques. Lors de la migration vers le Web aux alentours de 1995, les fonctions de recherche existantes dans le catalogue traditionnel furent simplement converties pour l'Internet (Lombardo et Condic, 200o). Bien qu'il soit disponible en tout temps et n'importe où, ce qui est apprécié par les chercheurs, nous constatons aujourd'hui que les fonctions du catalogue n'ont pas réellement évolué durant les 20 dernières années (Borgman, 1996 : 493). De façon simpliste, les catalogues offrent tous un processus de recherche débutant par la sélection d'un index de recherche, ce qui implique que l'utilisateur comprend bien de quoi il est question et à quoi servent ces index. Cette étape critique est suivie de l'entrée de mots-clés qui doivent se retrouver exactement dans l'index choisi pour ensuite générer une liste de résultats triée par date de publication.

Nous verrons que le catalogue traditionnel ne correspond plus aux attentes des chercheurs d'aujourd'hui, habitués aux moteurs de recherche Web qui offrent systématiquement une évaluation de la pertinence des résultats, la correction automatique des motsclés, et des suggestions de pistes de recherche.

\section{Les attentes des chercheurs d'aujourd'hui}

Observant la loi du moindre effort (Bates, 2003), les nouvelles générations de chercheurs s'attendent à des outils toujours accessibles et facilement utilisables sans formation préalable. Le chercheur d'aujourd'hui a droit à un large éventail d'outils de recherche et, s'il doit apprendre à les utiliser, il optera pour ceux qui le dispenseront d'apprentissage. La facilité est une donnée incontournable dans un contexte ou plusieurs services offrent la même information et des résultats équivalents, sinon similaires. En pratique, le catalogue devient plus facile à utiliser en offrant l'évaluation de la pertinence d'informations provenant de multiples sources intégrées, la correction automatique et des suggestions de mots-clés. Des fonctions du Web 2.o peuvent également être intégrées, offrant à l'utilisateur la possibilité d'ajouter du contenu aux notices bibliographiques et aux bases de données existantes.

L'entreprise Google a démontré qu'une interface simple et conviviale peut masquer des fonctions puissantes et complexes et son succès est ancré dans l'évaluation de la pertinence des résultats basés sur la crédibilité de chaque site Web. En moins de 10 ans, Google est devenu le mastodonte de l'information en ligne et,

\footnotetext{
4. http://www.slideshare.net/stoub/book-discovery-in-mass-digitizedenvironment/
} 
par conséquent, les chercheurs d'aujourd'hui s'attendent à ce que les catalogues en ligne effectuent également une évaluation de la pertinence au lieu du traditionnel tri par date de publication. La philosophie de Google explique le haut niveau d'innovation démontré par l'entreprise qui offre, en plus de son moteur de recherche, une gamme de produits de gestion d'information, tels Gmail, Google Documents, Picasa, Google Scholar.

"Google fait partie des quelques grandes entreprises qui ont compris une des règles fondamentales de l'Internet: il en coûte moins cher d'essayer quelque chose que de décider si on devrait l'essayer. ${ }^{5}$

(Don Marti, LinuxWorld, 13 Décembre 2007)

Le système doit également faciliter l'entrée de motsclés en offrant une correction d'orthographe et des suggestions automatiques permettant de chercher dans de multiples sources d'information intégrées. Révolue est l'époque où l'on pouvait demander aux utilisateurs de chercher et d'identifier une base de données prometteuse avant de débuter une recherche d'information. Ces frontières artificielles ne servent que des intérêts commerciaux et n'ont pas d'utilité pour le chercheur. De façon transparente, une liste de résultats doit donc pouvoir offrir de l'information provenant de divers éditeurs de contenus et sous divers formats (monographies, articles, images, sons, etc.).

Enfin, les attentes reliées au Web 2.0 peuvent se résumer à la possibilité pour l'utilisateur d'ajouter du contenu au catalogue, comme des commentaires, des critiques, des termes d'indexation et des "tags", mais habituellement ce ne sont pas ces données qui organisent l'information offerte par le site. L'impact relatif de cette nouvelle version du Web est décrite par Rory Litwin (May 6, 2007) qui suggère que les sites 2.0 de recherche de monographies (par exemple Amazon. com, et LibraryThing.com) offrent un contenu riche, préalablement organisé par des professionnels de l'information. Ainsi, les ajouts des utilisateurs sont une façon d'annoter permettant ensuite de filtrer, mais leur absence n'empêcherait pas ces services d'exister.

\section{Et celles de demain ...}

La nouvelle vague de moteurs de recherche Web expérimentaux suggère que la prochaine génération d'outils sera moins textuelle et plus visuelle. De nombreux sites, tels que grokker.com, tianamo.com, kartoo.com, zoomii.com, offrent maintenant une alternative visuelle à la longue liste linéaire de résultats textuels. Bien que le domaine de la visualisation de l'information en soit encore à ses débuts, il y a fort à parier que les outils de recherche subiront une évolution semblable à celle des systèmes d'exploitation. Rappelons-nous qu'il

5. Traduction de l'auteur.

\section{La nouvelle vague de moteurs de recherche Web expérimentaux suggère que la prochaine génération d'outils sera moins textuelle et plus visuelle.}

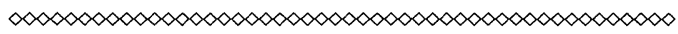

y a moins de 20 ans tous les ordinateurs personnels (sauf Apple) n'offraient que des interfaces textuelles de type DOS ou Unix et que l'utilisateur devait connaître exactement une série de commandes prédéterminées. Les outils de recherche actuels ressemblent à ce modèle d'interaction puisqu'ils ne sont que textuels et demandent des mots-clés (comparables à une commande) reconnus par le système avant de retourner des résultats purement textuels.

\section{Un outil qui ne correspond plus aux attentes des chercheurs}

On relève des problèmes de conception d'interface (Mi et Weng, 2008) qui rendent l'utilisation du catalogue difficile sans formation spécifique. Il y a, en effet, un contraste marqué entre l'unique boîte à mots-clés de Google et les sélections multiples offertes par les catalogues traditionnels. La conception des catalogues reflète l'époque où de nombreux paramètres de recherche étaient utilisés par des chercheurs ayant reçu une formation spécifique et/ou ayant accès à du personnel expérimenté à la bibliothèque même où ils s'étaient rendus. Même les interfaces de recherche " simples " sont souvent surchargées de jargon (par exemple, "index", "Dewey ", "vedette-matière », etc.) dont il faut connaître la signification pour choisir l'index de recherche et les clés d'accès appropriées au type de recherche à mener. La loi du moindre effort conduira l'utilisateur néophyte à utiliser les options par défaut sans comprendre l'impact de ce choix sur les résultats obtenus. Par exemple, l'index " Titre " produira systématiquement moins de résultats potentiellement pertinents comparativement à l'index " Tous les champs ». L'utilisateur du premier index aura l'impression que la bibliothèque possède peu de documents, parfois très appropriés et l'utilisateur du deuxième index aura l'impression d'une collection plus vaste mais moins pertinente à ses besoins.

L'analyse des résultats de recherches faite dans des catalogues révèle qu'entre $35 \%$ et $50 \%$ des recherches (Markey, 1986) offrent le message " Aucun document retrouvé », suggérant à l'utilisateur que la bibliothèque n’a pas de matériel traitant du sujet spécifié (Bates, 2003:7). Ce fort taux d'échec s'explique en grande partie par les fautes d'orthographe à la saisie et/ou un vocabulaire déficient dans un nouveau champ de connaissances (Larson, 1991 : 208). En effet, la conception du catalogue traditionnel force l'utilisateur à deviner et 


\section{Afin de sauver le catalogue, un changement profond est nécessaire et on ne peut plus se contenter de modifications cosmétiques.}

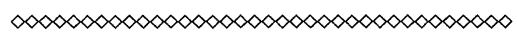

à épeler correctement au clavier un ou plusieurs mots présents dans la notice cible. L'échec d'une requête est donc assuré s'il y a faute de frappe ou si la notice cible contient un vocabulaire moins connu.

De plus, nous savons depuis le début des années 1980 que les catalogues en ligne sont difficiles à utiliser pour effectuer une recherche par sujet (Bates, $2003: 49$; Markey, 2007:2). Bien que le vocabulaire des vedettes-matières soit parfois peu familier, les analyses des requêtes entrées dans le catalogue démontrent que dans $74 \%$ des cas (Carlyle, 1989 : 44), les mots-clés saisis se retrouvent dans les vedettes-matières. Il y a donc un travail à faire pour augmenter la visibilité des vedettesmatières et de leurs relations génériques, spécifiques et associées.

Enfin, suivant toujours la loi du moindre effort, la syntaxe de recherche booléenne est difficile à apprendre et peu utilisée. De nombreuses études (Borgman, 1986 : 392) ont relevé chez les chercheurs des difficultés persistantes à comprendre la logique Booléenne. Jansen \& Pooch (2001) rapportent que moins de $10 \%$ des requêtes font usage d'opérateurs booléens.

Pendant ce temps, les chercheurs de livres se tournent vers d'autres sources offrant des services équivalents (par exemple : amazon.com ; librarything. com ; bookswim.com ; zoomii.com) à l'aide d'interfaces attrayantes et en constante évolution. Force est de constater que :

\section{«Laposition du catalogue en ligne dansle marché de la recherche s'est érodée jusqu'au point où l'on se demande si cet outil peut encore attirer l'at- tention des chercheurs d'information. ${ }^{6}$ \\ (Calhoun. 2006:10)}

Afin de sauver le catalogue, un changement profond est nécessaire et on ne peut plus se contenter de modifications cosmétiques, ce que Roy Tennant (2005) décrit familièrement comme le "maquillage d'un cochon ». Comment les modules d'exploitation des catalogues proposés par les fournisseurs de SIGB en sont-ils arrivés là et que font ces mêmes fournisseurs pour aider les bibliothèques à s'en sortir ? La prochaine section décrit la situation de l'industrie des systèmes intégrés de gestion de bibliothèque et démontre que, pour des raisons d'ordre commerciales et techniques, les fournis-

6. Traduction de l'auteur. seurs n'ont pas su répondre aux chercheurs demandant un catalogue de la nouvelle génération.

\section{Les systèmes intégrés de gestion de bibliothèque (SIGB)}

Le marché des SIGB est mature (Sero Consulting Ltd., 2008) et fait face à des revenus stables ou réduits, ce qui constitue un contexte favorable pour les multiples acquisitions et fusions ${ }^{7}$. Ce marché est donc concentré entre quelques gros fournisseurs offrant des produits très similaires, forçant une différenciation par le prix et une vulnérabilité commerciale additionnelle. Par exemple, le récent rapport produit par une firme de consultants indépendants pour le gouvernement britannique (Sero Consulting Ltd., 2008) démontre que $90 \%$ du marché des SIGB anglais est concentré entre quatre fournisseurs. Cette situation devient d'autant plus inquiétante lorsqu'on apprend que la moitié d'entre eux appartiennent à des sociétés à capitaux privés ayant comme premier mandat d'augmenter la valeur de revente de leurs holdings. Dans un tel contexte, il est difficile de prédire quels fournisseurs et quels produits seront disponibles l'année prochaine, et le rapport suggère clairement de ne pas acheter de SIGB pour l'instant.

$\mathrm{Au}$ cours d'une communication faite en octobre $2007^{8}$, Carl Grant, un haut dirigeant de l'industrie des SIGB totalisant plus de 30 ans d'expérience, a présenté plusieurs éléments illustrant la position actuelle des éditeurs. Selon lui, le cycle de vente d'un SIGB demande environ deux ans et n'implique généralement que l'éditeur du SIGB et la direction de la bibliothèque. Les éditeurs de SIGB doivent donc augmenter leurs prix afin de récupérer les frais de vente. Ils ont intérêt à investir dans les fonctions internes de la bibliothèque (prêt, catalogage, achats, etc.), au détriment du module de recherche offert au public, souvent secondaire dans la décision d'achat. Carl Grant admet que les catalogues actuels sont de piètre qualité tout en affirmant que les bibliothèques ont créé cette situation en demandant beaucoup plus de peaufinage des fonctions existantes au détriment des changements de fond. Cette affirmation a également été faite en 2006 par Pat Sommers ${ }^{9}$, président de Sirsi/Dynix, qui affirmait que son entreprise dépensait plus de 10 millions de dollars par an pour répondre aux demandes d'amélioration des fonctions de gestion existantes, et que par conséquent, il ne lui restait plus beaucoup d'argent pour développer de meilleurs outils de recherche.

La complexité des SIGB permet difficilement à leur éditeur d'effectuer des changements de fond. Un système intégré offre l'efficacité et l'efficience de la gestion de l'information en imposant des processus

\footnotetext{
7. Pour suivre l'évolution des acquisitions et fusions des éditeurs de SIGB, consulter le site : http://www.librarytechnology.org/automationhistory. pl?SID $=20080703935791015$.

8. http://incolntrail.typepad.com/ilssymposium/2007/10/podcast-carl--1.html.

9. http://www.oclc.org/ca/en/memberscouncil/meetings/2006/default.htm, voir présentations de février.
} 
normalisés dans la gestion des bibliothèques (acquisition, gestion de la collection, catalogage, recherche, prêt, etc.). Cette normalisation empêche la flexibilité locale de chaque fonction pour s'adapter aux demandes des utilisateurs spécifiques puisqu'un changement dans un système intégré aura un grand impact sur tout le traitement de l'information. De plus, la distribution d'une version modifiée impose à l'éditeur la gestion de multiples versions du SIGB, ce qui rend les mises à jour et le support de plus en plus dispendieux. Par exemple, Carl Grant répertorie 342 façons d'effectuer des achats et Pat Sommers décrit de multiples conditions permettant à l'utilisateur de réserver un livre. Il est difficile d'assurer la stabilité d'une telle application puisque, pour s'adapter à cette diversité, elle doit offrir un millier de paramètres à définir pour chaque client, ce qui rend impossible la tâche de tester une mise à jour avec toutes les configurations possibles.

Le catalogue en ligne comme outil de recherche se trouve donc dans la position où il devient de moins en moins pertinent et les solutions novatrices ne peuvent provenir rapidement des éditeurs de SIGB. Depuis un peu plus de deux ans, quelques bibliothèques ont cependant démontré qu'il est possible de réinventer le catalogue et de réanimer l'intérêt du public pour les services de la bibliothèque.

\section{Des catalogues novateurs}

Nous exposons trois applications représentatives d'une nouvelle génération de catalogues. Ces outils de recherche sont tous indépendants du SIGB, ce qui leur permet d'être en constante évolution selon l'agenda de la bibliothèque. En second lieu, nous portons une attention particulière à la jeune et dynamique communauté $\mathrm{du}$ code source libre pour bibliothèques, laquelle fait beaucoup parler d'elle depuis peu. Enfin, nous évaluons la réponse des grands éditeurs de SIGB à ces profonds changements du catalogue.

\section{Le catalogue indépendant du SIGB}

L'implantation des trois catalogues novateurs qui seront décrits fut entérinée par des instances décisionnelles et guidée par les deux principes suivants : premièrement, le changement est plus important que la perfection et, deuxièmement, il faut mieux contrôler le catalogue public en le rendant indépendant du SIGB. Le premier principe suggère que la perte de certaines fonctions, ou même de données, doit être acceptée afin de permettre un changement radical et rapide.

Ce type de changement plutôt radical est possible si on accepte l'idée que l'outil de recherche intégré au SIGB ne sera plus ou sera beaucoup moins utilisé par le public. En pratique, cette démarche implique qu'initialement toutes les notices bibliographiques du SIGB seront copiées vers un système de requête indépendant qui, par la suite, recevra périodiquement les chan- gements et ajouts ainsi que les données de prêt. Ces données étant maintenant libérées du SIGB, le nouveau catalogue peut être développé à l'interne ou par un fournisseur de services. Ce modèle permet une évolution rapide et personnalisée du catalogue puisque la bibliothèque n'est plus dépendante des ressources et du calendrier de développement du fournisseur de son SIGB.

C'est ce modèle d'architecture technique qui fut d'abord démontré par le catalogue Endeca offert par les bibliothèques de l'Université de la Caroline du Nord et ensuite par les centaines d'implantations du catalogue Aquabrowser. Quant au catalogue de l'Université de l'État de Washington, il est non seulement indépendant du SIGB, mais également hébergé chez OCLC, qui devient donc responsable de son bon fonctionnement.

\section{Une pionnière : l'Université de la Caroline du Nord ( $\mathrm{NCSU}^{10}$ )}

Le 2 janvier 2006, les bibliothèques de la NCSU sont les premières à offrir un catalogue novateur (Figure 1), démontrant ainsi qu'il est possible d'effectuer un changement de fond et de rajeunir l'image de la bibliothèque. Cet outil de recherche a reçu beaucoup d'attention médiatique et il marque le début d'une nouvelle génération de catalogues. À la NCSU, les données bibliographiques gérées par le SIGB sont exportées vers le moteur d'indexation et de requête Endeca, qui nourrit ensuite une interface Web développée à l'interne.

Ce catalogue s'efforce notamment de réduire le nombre de requêtes infructueuses en utilisant les radicaux $^{11}$ et la correction d'orthographe afin de générer des suggestions et de produire une liste des meilleurs résultats triée en ordre de pertinence. La Figure 1 montre que la requête "globla warming » devient automatiquement "global warming".

L'algorithme d'évaluation de la pertinence est basé sur plusieurs critères pondérés selon une évaluation périodique des résultats qu'il génère. La définition précise de cet algorithme n'est pas disponible, mais nous savons que NCSU considère les éléments suivants ${ }^{12}$ :

- le nombre d'occurrences du mot cherché divisé par le nombre de mots dans la notice. Ce pourcentage doit être normalisé afin de ne pas favoriser systématiquement les notices courtes ;

- la présence d'un mot-clé dans certains champs (par exemple : les champs titre ou vedettesmatière) est plus significative que dans les autres (par exemple : le champ résumé) ;

- la popularité du document basée sur les données de prêt ;

- la date de publication du document.

10. En anglais : North Carolina State University.

11. Nous faisons référence ici à l'extraction automatique de mots-racine ex.: amoureux et amoureuse ont le radical commun "amour ".

12. http://wwwnew.lib.ncsu.edu/staff/kaantelm/2007-NCserials.ppt. 
Figure 1

Catalogue de l'Université de la Caroline du Nord (http://www.lib.ncsu.edu/catalog/)

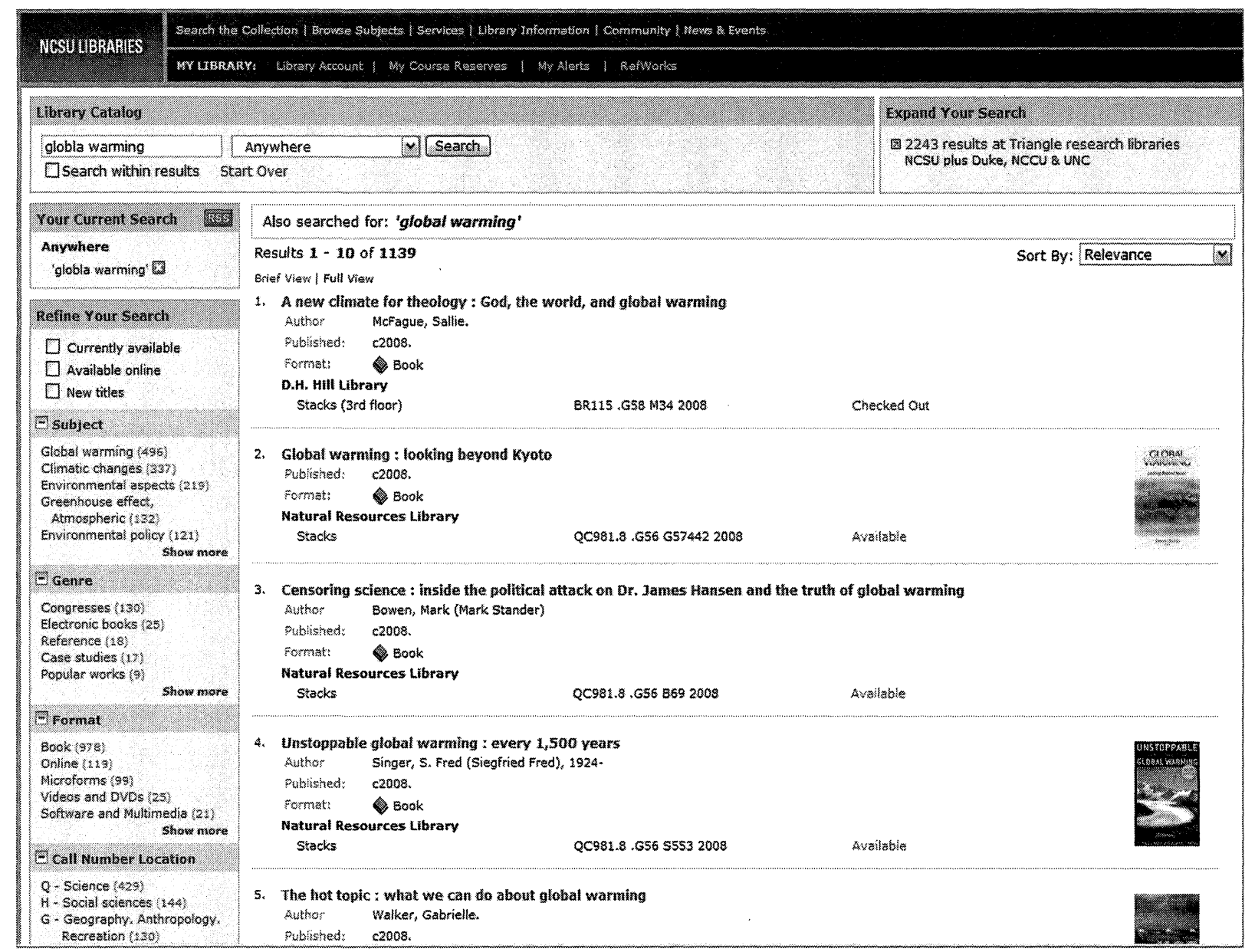

De plus, ce catalogue offre de nombreuses facettes (panneau de gauche, Figure 1) permettant de raffiner progressivement les résultats de la recherche en conjonction avec les mots-clés. Par exemple, le chercheur peut limiter ses résultats par format (par exemple : seulement les monographies), par vedettes-matières retrouvées dans les résultats, par la période historique, par genre (par exemple : fiction), etc. Ce catalogue semble également être le seul à tenter de capitaliser sur l'information implicite contenue dans l'indice de la Classification de la Library of Congress (LCC), assigné à chaque document, en permettant de raffiner une recherche selon le domaine auquel l'ouvrage se rapporte (par exemple : sciences ou agriculture).

Les responsables des outils du réseau de bibliothèques de la NCSU ont voulu mesurer l'avantage du nouveau catalogue comparativement à leur catalogue traditionnel. On a demandé à deux groupes de cinq étudiants inscrits au baccalauréat d'effectuer dix tâches prédéfinies, incluant la localisation de ressources spécifiques (par exemple : trouver un titre ou un auteur) et des recherches concernant des sujets plus abstraits (par exemple : trouver cinq livres traitant des maladies affectant les chiens). Les mesures d'efficacité indiquent que les deux catalogues permettent d'accomplir les tâches, mais le nouveau catalogue est perçu comme étant plus facile à utiliser et demande significativement moins de temps. Il est à noter que cette conception expérimentale ne peut être considérée statistiquement probante en raison de son petit nombre de participants et qu'il est possible que la différence entre les systèmes soit en fait une différence préexistante entre la capacité cognitive des deux groupes.

\section{Le plus répandu : Aquabrowser}

Implanté dans plus de 250 bibliothèques en Europe et aux États-Unis, le catalogue Aquabrowser ${ }^{13}$ de l'entreprise Medialab s'est hissé au premier rang du marché des catalogues de nouvelle génération. Medialab entretient des relations serrées avec le monde des bibliothèques et l'image de ce catalogue indépendant du SIGB est grandement définie par son interface de furetage visuel unique et novatrice (panneau de gauche, Figure 2). Le chercheur est d'abord invité à saisir un ou plusieurs mots-clés. Cette requête est affichée au centre d'une " constellation " d'environ 20 mots qui co-occurrent 


\section{Figure 2}

Catalogue de la bibliothèque de l'Université de l'0klahoma (http://boss.library.okstate.edu/)

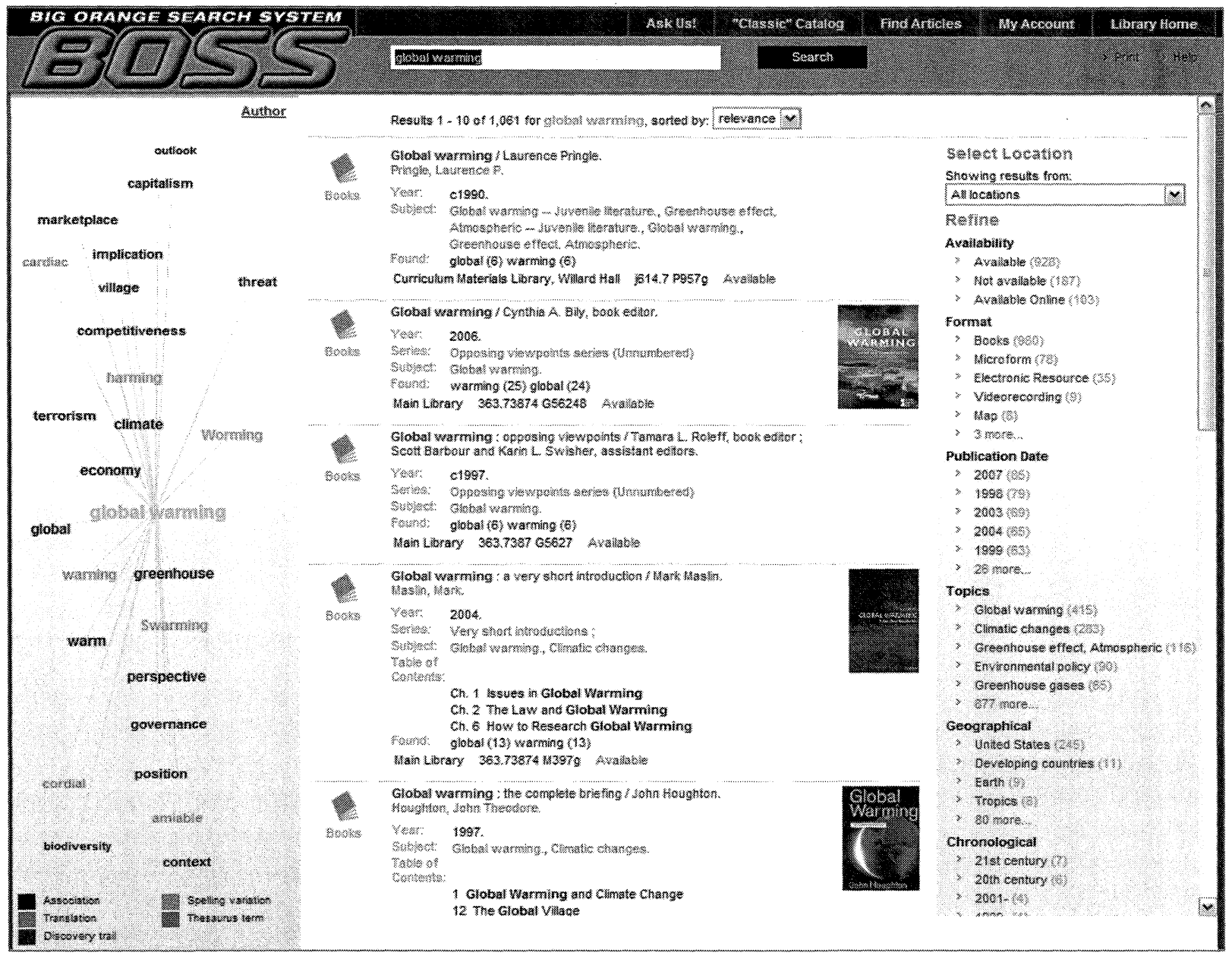

souvent avec les mots spécifiés au sein des notices bibliographiques.

Dans la Figure 2, la constellation générée par le terme " global warming" affiche les termes " greenhouse ", " climate ", « threat ", etc. Le chercheur peut sélectionner un de ces termes afin d'inspecter la liste de notices qui y sont associées ou raffiner sa requête initiale en y ajoutant un des termes suggérés. Cette interface de furetage sémantique tente donc d'alléger l'effort mental requis pour effectuer une recherche en offrant à l'utilisateur un outil convivial permettant de reconnaître et de sélectionner des termes valides dans le contexte d'une collection, lui évitant d'avoir à les générer de mémoire ou en inspectant des notices bibliographiques déjà repêchées. Aquabrowser offre également l'évaluation de la pertinence, de nombreuses facettes permettant de raffiner dynamiquement une recherche (panneau de droite, Figure 2) ainsi que la correction automatique de l'orthographe.

Après avoir considéré divers catalogues de nouvelle génération, le réseau des bibliothèques de l'Université de l'Oklahoma aux États-Unis a choisi d'implanter une version du catalogue Aquabrowser, offerte depuis l'automne 2007. Guidée par le principe que le change- ment est plus important que la perfection ${ }^{14}$, l'implantation du nouveau catalogue demanda environ un an et l'outil est en évolution constante. Malgré la disponibilité de l'ancien catalogue, deux mois après la mise en opération d'une nouvelle interface, les deux tiers des recherches étaient déjà effectuées à l'aide d'Aquabrowser, démontrant une adoption rapide du nouvel outil par la majorité des utilisateurs.

La recherche semble également plus fructueuse puisque le nombre de requêtes sans résultats est passé à $5 \%$, comparativement à $38 \%$ avec l'ancien catalogue. Quand les facettes sont utilisées pour raffiner la recherche, le format (par exemple: monographies seulement) est choisi à $24 \%$ du temps et les vedettes-matières le sont à $23 \%$. Il s'avère également que le tri par pertinence offert par défaut est modifié dans $2 \%$ des cas seulement. Il ne fait pas de doute que la loi du moindre effort influence ici encore le comportement de l'utilisateur, mais les gestionnaires croient aussi que, dans la majorité des cas, l'évaluation automatique de la pertinence produit des résultats d'une qualité suffisante et

\footnotetext{
14. http://www.slideshare.net/youthelectronix/prestamo/
} 
Figure 3

Site des bibliothèques de l'Université de l'État de Washington (http://www.lib.washington.edu/)

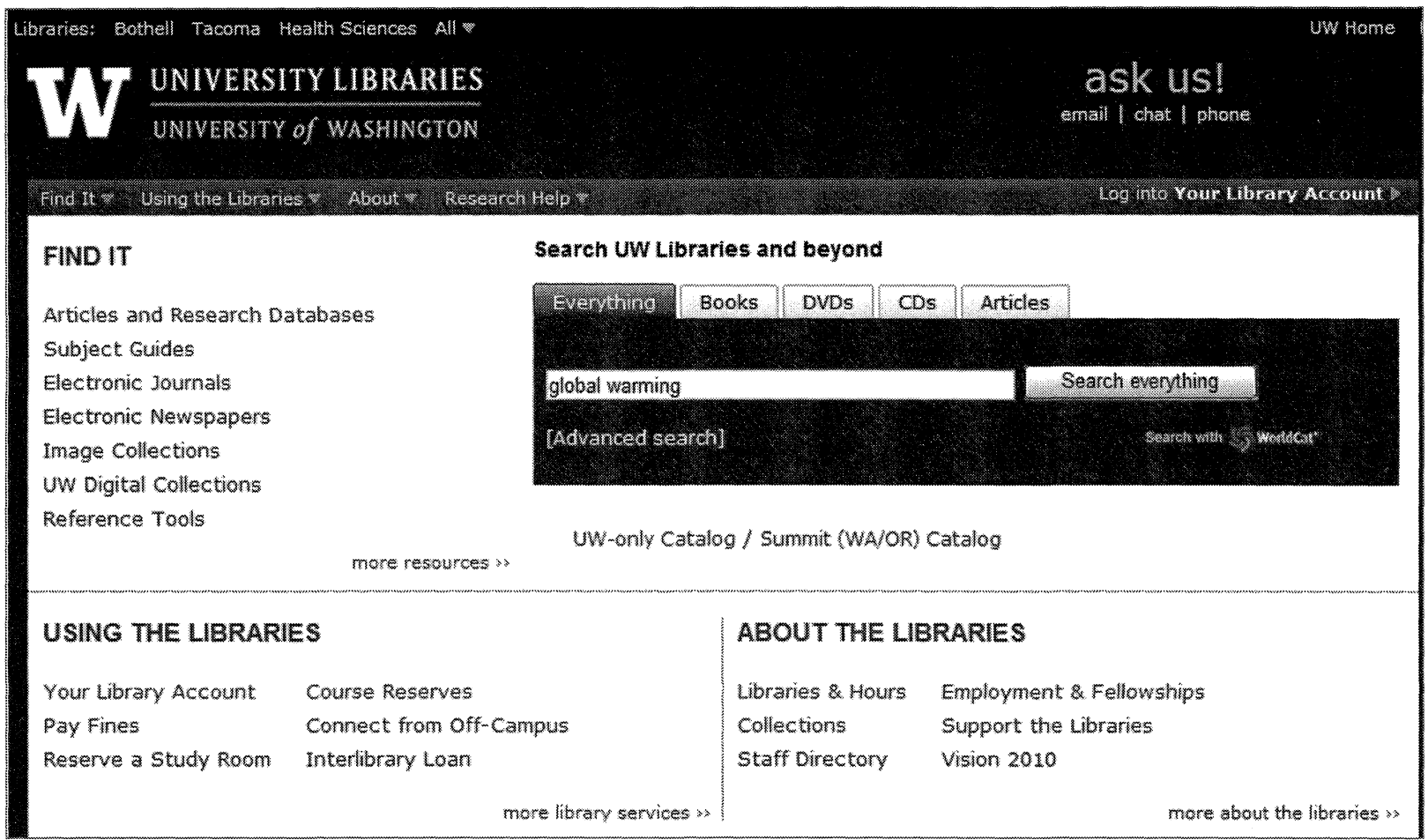

que le chercheur ne sent pas le besoin d'essayer d'autres options de tri (par exemple : par date de publication).

\section{Le catalogue hébergé : OCLC Worldcat Local}

Le catalogue novateur de l'Université de Washington ${ }^{15}$ est hébergé chez OCLC, ce qui donne aux chercheurs un accès direct à une impressionnante richesse d'information et les avantages d'une conversion FRBR $^{16}$. Pour l'utilisateur, l'avantage principal du FRBR est la production d'une liste de résultats plus courte et plus précise. Par exemple, dans un catalogue traditionnel, une recherche dans l'index « titre " pour le mot-clé "Hamlet » ramènera plusieurs centaines d'éditions, de traductions et de critiques de l'ouvrage, et il sera difficile de localiser la pièce de Shakespeare en version originale. La même recherche effectuée dans un catalogue $\mathrm{FRBR}^{17}$ produit une liste de résultats beaucoup plus courte, proposant un seul titre, soit « Hamlet, de William Shakespeare ». À la suite de la sélection de cet ouvrage générique, le catalogue offre une fiche détaillée incluant les diverses éditions et traductions de l'ouvrage.

Le catalogue WorldCat Local est une application hébergée, ce qui veut dire qu'elle est physiquement installée sur un serveur situé chez OCLC qui devient alors responsable de son bon fonctionnement. La bibliothèque n'a qu'à intégrer à son site Web les liens poin-

15. http://www.lib.washington.edu/

16. Functional Requirements for Bibliographic Records.

17. Voir l'exemple de catalogue FRBR chez http://fictionfinder.oclc.org/ tant vers son catalogue. Ce modèle de fonctionnement offre les avantages du catalogue indépendant du SIGB et il réduit la charge de travail des employés de la bibliothèque qui n'ont pas à gérer d'infrastructures technologiques. Par contre, la bibliothèque a moins de contrôle sur la conception et l'apparence de son catalogue.

Le site Web des bibliothèques de l'Université de Washington est un bon exemple de conception offrant une page principale, simple et concise, qui facilite les tâches les plus fréquentes sans surcharger d'information les utilisateurs. La boîte de recherche par mots-clés est clairement offerte dès la page principale (Figure 3) puisqu'il est probable qu'une grande partie des utilisateurs ayant navigué jusqu'à son site désirent utiliser cet outil.

Comme la plupart des catalogues de nouvelle génération, WorldCat Local offre également des facettes permettant de raffiner la recherche, d'utiliser la correction automatique et les suggestions. De plus, l'évaluation de la pertinence est unique puisqu'elle considère que, tous les autres critères étant égaux, les ouvrages disponibles dans la bibliothèque locale du chercheur seront jugés les plus pertinents, suivis de ceux qui sont disponibles dans le réseau de l'université, de ceux qui sont disponibles par prêts entre bibliothèques et enfin, de ceux qui appartiennent à d'autres bibliothèques membres du consortium mondial d'OCLC. Ainsi, la possibilité pour une requête de ne produire aucun résultat est réduite. Même les requêtes les plus obscures ont de bonnes chances de succès puisqu'elles sont menées dans une collection qui inclut tous les docu- 
ments appartenant à des milliers de bibliothèques à travers le monde.

De plus, le catalogue WorldCat Local offre une incomparable richesse de contenu incluant 30 millions d'articles en ligne intégrés à la collection de la bibliothèque. On abat ainsi la frontière artificielle qui existe encore entre les articles de périodiques et les types de documents traditionnellement décrits et repérables au catalogue (monographies, documents audio-visuels, etc.). Les notices bibliographiques proposent souvent un ou plusieurs résumés, une table des matières, des évaluations critiques et la possibilité pour l'utilisateur d'ajouter ses propres notes et impressions.

L'impact du nouveau catalogue tel que décrit par les représentants des bibliothèques de l'Université de Washington $^{18}$ est impressionnant. De façon générale, l'interface de recherche est simplifiée et englobe des services auparavant indépendants, ce qui permet d'offrir aux chercheurs un seul outil de recherche convivial donnant accès à un contenu très enrichi. Il faut maintenant beaucoup moins d'étapes pour effectuer une demande de prêt intra-réseau ou entre institutions. Seulement six mois après le lancement du nouveau catalogue, les demandes intra-réseau ont augmenté de $65 \%$ et de $70 \%$ entre institutions. Ceci démontre qu'une technologie facilitant l'accès aux services peut faire augmenter la demande simplement parce que l'on a aplani les barrières technologiques.

\section{Autres catalogues novateurs}

Les catalogues auxquels nous faisons maintenant référence sont décrits succinctement et, seules, leurs caractéristiques novatrices sont soulignées. Le catalogue de furetage East 41 st Street ${ }^{19}$ est un excellent exemple d'utilisation des vedettes-matières et de recherche par mots-clés en parfaite intégration, cela permet de raffiner la recherche par sujet de façon dynamique et intuitive. La bibliothèque publique d'Edmonton ${ }^{20}$ offre une boîte de recherche dès la première page d'un site attrayant qui inclut un " nuage de mots " présentant les termes qui se retrouvent souvent dans le catalogue. Le catalogue donne ainsi un aperçu global des sujets les plus présents dans la collection et fournit en même temps un vocabulaire de requête pertinent en contexte.

Peel's Prairie Provinces ${ }^{21}$ donne accès à une collection spécialisée en histoire et culture des Prairies canadiennes à l'aide d'un catalogue novateur, même s'il est parfois surchargé. Celui-ci offre à l'utilisateur de multiples éléments d'information interactifs décrivant l'ensemble des résultats d'une requête tels que la distribution des dates de publication, les emplacements géographiques associés aux ouvrages ainsi que des nuages de

18. http://gslis.simmons.edu/podcasts/index.php?id=47

19. http://www.amitgupta.info/E41ST/RIA/E 41 ST.html

20. http://www.epl.ca/EPLCloudNine.cfm?all=yes

21. http://peel.library.ualberta.ca/index.html
Bien que le domaine des SIGB libres soit actuellement très dynamique, ces produits représentent une minuscule part du marché.

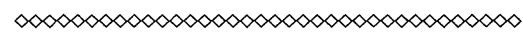

mots qui décrivent la distribution des langues et des sujets.

Enfin, la bibliothèque publique de Phoenix ${ }^{22}$, en Arizona, innove en donnant la possibilité de raffiner une recherche sur la base de la fréquence d'emprunt des ouvrages, sur le statut de "Best Sellers", sur les recommandations des employés de la bibliothèque, sur les récipiendaires de prix, etc.

\section{Les solutions « logiciels libres »}

L'État américain de la Géorgie a démontré qu'il est possible de développer un SIGB libre pour un grand réseau de bibliothèques. La solution Evergreen ${ }^{23}$ est supportée par l'entreprise de consultation Equinox et permet de gérer toutes les opérations de la bibliothèque, à l'exception des achats et des périodiques. De façon générale, ces applications sont des versions libres de SIGB commerciaux qui se différencient par un prix d'achat imbattable : elles sont distribuées gratuitement ! La bibliothèque qui choisit une solution libre peut ensuite en faire ce qu'elle veut et se libère ainsi des intérêts et échéanciers d'un fournisseur commercial. Le modèle d'affaire du logiciel libre repose sur la vente de services offerts, dans le meilleur des cas, par une communauté de développeurs experts. Ce modèle permet à la bibliothèque de préciser ce qu'elle veut effectuer comme changements à l'application et de choisir ensuite le développeur capable de faire le travail à moindre coût.

Selon Marshall Breeding ${ }^{24}$, l'expérience américaine a démontré que la solution libre n'est pas nécessairement moins coûteuse qu'un produit commercial. Chaque nouvelle implantation d'un SIGB libre demande un investissement significatif afin de modifier la solution développée pour un autre contexte organisationnel. Ce genre de projet demande également l'embauche et la rétention de ressources humaines spécialisées et implique un budget de développement et une période de test et de rodage difficiles à estimer et à planifier.

De plus, bien que le domaine des SIGB libres soit actuellement très dynamique, ces produits représentent une minuscule part du marché et sont implantés surtout dans les petites bibliothèques publiques. Cette situation pourrait bientôt changer puisque que les universités

22. http://www.phoenixpubliclibrary.org/index.jsp

23. http://gapines.org/opac/en-US/skin/default/xml/index.xml

24. http://www.oclc.org/programsandresearch/dss/default.htm. 


\section{Le changement doit être une priorité, la perfection n'est pas possible et elle ralentit l'évolution.}

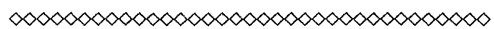

Laurentienne de Sudbury et McMaster de Hamilton, en Ontario, le réseau des bibliothèques publiques de la Colombie-Britannique, l'État de la Géorgie et Equinox se sont associés afin de développer des modules d'achats et de gestion des périodiques. De plus, le réseau de la Colombie-Britannique déploie présentement sa version de la solution Evergreen à travers la province.

Il existe également d'autres solutions libres distribuées à partir des États-Unis. Le SIGB libre Koha ${ }^{25}$ s'adresse aux bibliothèques individuelles ou à de petits réseaux. Scriblio ${ }^{26}$ est développé par l'Université du New Hampshire, à Plymouth, et VuFind ${ }^{27}$ par l'Université Villanova en Pennsylvanie. Le moteur d'indexation libre Solr ${ }^{28}$ est de plus en plus utilisé comme moteur de recherche qui reçoit et indexe les extraits de données du SIGB et fournit ensuite l'interface Web d'un catalogue développé spécifiquement pour la bibliothèque.

\section{La réponse des éditeurs de SIGB commerciaux}

Quelques éditeurs de SIGB commerciaux ont réagi aux initiatives de catalogues de nouvelle génération et offrent maintenant de nouveaux modules de recherche intégrés au SIGB. L'éditeur du système Ex Libris propose l'outil de recherche Primo, implanté notamment à l'Université du Minnesota ${ }^{29}$, qui offre l'évaluation de la pertinence, les facettes permettant de raffiner la recherche ainsi que la correction automatique et les suggestions. Implanté à la bibliothèque de l'État de la Tasmanie ${ }^{30}$, l'éditeur du système Talis démontre sa nouvelle interface de recherche appelée Talis Plus, laquelle offre sensiblement les mêmes fonctions que Ex Libris Primo, malgré une interface esthétiquement différente. Enfin, Innovative Interfaces a implanté son catalogue Encore dans les bibliothèques de l'Université de la Californie à Fresno $^{31}$.

Ces produits, maintenant disponibles chez les grands éditeurs de SIGB, démontrent que la nouvelle génération de catalogues en ligne se distingue en incluant systématiquement l'évaluation de la pertinence, les facettes permettant de raffiner la recherche et

\footnotetext{
25. http://liblime.com/koha.

26. http://about.scriblio.net/about et http://library.plymouth.edu/

27. http://www.library.villanova.edu/

28. http://lucene.apache.org/solr/

29. http://prime7.oit.umn.edu:1701/primo_library/libweb/

30. http://www.statelibrary.tas.gov.au/

31. http://www.csufresno.edu/library/
}

la correction automatique des mots-clés et des suggestions de recherche. Mais les grands éditeurs de SIGB n'ont pas été les instigateurs de cette nouvelle génération de catalogue et malgré leurs ressources considérables, leurs produits semblent pour l'instant relever davantage de l'imitation que de l'innovation. Les institutions qui prévoient acheter un catalogue intégré au SIGB peuvent se poser les questions suivantes :

- dans un contexte où le rythme effréné de l'évolution des technologies reliées à l'Internet réduit considérablement la durée de vie d'une génération d'outils de recherche, une bibliothèque peuelle espérer qu'un fournisseur de SIGB puisse faire évoluer le catalogue au rythme des attentes des chercheurs?

- véhiculée par son catalogue, l'image des services en ligne de la bibliothèque devrait-elle être contrôlée par un fournisseur qui doit concilier de multiples intérêts et satisfaire une multitude de clients qui utilisent divers modules (achats, catalogage, prêt, etc.) de façon distincte, tout en s'assurant d'un retour acceptable pour ses investisseurs?

\section{Conclusions}

Nous avons tenté de démontrer que les catalogues de bibliothèques en ligne traditionnels ne peuvent plus se mesurer aux multiples outils de recherche d'information novateurs, attrayants et dynamiques offerts sur le Web. La description des catalogues de nouvelle génération Endeca, Aquabrowser et OCLC Worldcat Local révèle qu'il est possible de réinventer cet outil en le rendant indépendant du système intégré de gestion de bibliothèque et en priorisant le changement plutôt que la perfection. Cette situation est d'autant plus critique que "l'avenir de la bibliothèque universitaire repose sur un catalogue efficace. C'est une des tâches les plus pressantes à accomplir "32 (Mi et Weng, $2008: 18$ ).

À la lumière des informations et de la description des catalogues de nouvelle génération présentés dans cet article, il est possible d'émettre certaines recommandations pour ceux qui, en effectuant un changement radical, veulent tenter de freiner et même d'inverser la baisse d'utilisation du catalogue.

Le changement doit être une priorité, la perfection n'est pas possible et elle ralentit l'évolution. Afin de demeurer pertinent aux yeux des chercheurs, ayant accès à une variété grandissante d'outils novateurs de recherche, le catalogue, vitrine par excellence d'une bibliothèque sur le Web, doit être dynamique en offrant constamment de nouveaux services, quitte à éliminer ceux qui sont moins utilisés.

Accepter une solution commerciale intégrée ne permet pas toujours de contrôler l'image virtuelle de

32. Traduction de l'auteur 
la bibliothèque et donc de séparer l'outil de recherche offert au public des applications de gestion interne. Un catalogue souple et indépendant du SIGB s'adaptera plus facilement et rapidement aux attentes et aux comportements des chercheurs.

L'approche "logiciel libre », plutôt que l'implantation des solutions de type Endeca ou Aquabrowser, est envisageable si on détient les ressources pour retenir les services de développeurs spécialisés (soit à l'interne ou en consultation).

En somme, le catalogue réinventé implique un décloisonnement entre les outils de gestion interne de la bibliothèque et les outils de recherche d'information qu'elle présente à ses utilisateurs. L'efficacité d'un catalogue se mesure à sa capacité à repérer des ressources pertinentes dans une collection, alors que l'efficacité d'un SIGB se traduit, entre autres, par la capacité à fournir des données sur l'origine et l'utilisation de ces ressources. En réclamant des systèmes intégrés, les bibliothèques ont sans doute freiné la conception d'outils spécialisés adaptés et plus performants pour chacune des fonctions. Heureusement, de nouvelles initiatives indiquent qu'il est possible d'effectuer maintenant ce décloisonnement pour offrir de meilleurs services aux utilisateurs. $\odot$

\section{Sources consultées}

Bates, Marcia J. 2003. Improving user access to library catalog and portal information. Library of Congress. $58 \mathrm{p}$. http://www.loc. gov/catdir/bibcontrol/2.3BatesReport6-03.doc.pdf

Borgman, Christine L. 1986. Why are online catalogs hard to use ? Lessons learned from information-retrieval studies. Journal of the American Society for Information Science. 37(6) : 387-400.
Borgman, Christine L. 1996. Why are online catalogs still hard to use ? Journal of the American Society for Information Science. $47(7): 493-503$.

Calhoun, Karen. 2006. The changing nature of the catalog and its integration with other discovery tools. Rapport préparé pour la Library of Congress. 52 p. http://www.loc.gov/catdir/calhounreport-final.pdf

Carlyle, Allison. 1989. Matching LCSH and user vocabulary in the library catalog. Cataloging \& Classification Quarterly. 10(1-2): 37-63.

Eden, Brad. 2007. Reinventing the OPAC. Library Technology Reports. 43(6) : 13-40.

Jansen, Bernard J. and U. Pooch. 2001. A review of Web searching studies and a framework for future research. Journal of the American Society for Information Science and Technology. 52(3) : 235-246.

Larson, Ray R. 1991. The decline of subject searching : Long-term trends and patterns of index use in an online catalog. Journal of the American Society for Information Science. 42(3): 197-215.

Litwin, Rory. May 6, 2007. Why Web 2.o is Leading back to Full Cataloging. Library Juice blog. Accédée le 3 juillet, 2008, http://libraryjuicepress.com/blog/ ?p=256.

Lombardo, S. V. and K. S. Condic. 200o. Empowering users with a new online catalog. Library Hi Tech. 18(2) : 130-141.

Markey, Karen. 1986. Users and the online catalog: Subject access problems. In The Impact on Online Catalogs. J. R. Matthews. New York : Neal-Schuman. pp. 35-69.

Markey, Karen. 2007. The Online Library Catalog : Paradise Lost and Paradise Regained ? D-Lib. 13(1/2). Accédée le 28 août, 2008, http://www.dlib.org/dlib/januaryo7/markey/ormarkey. html

Mi, Jia and Cathy Weng. 2008. Revitalizing the Library OPAC: Interface, Searching, and Display Challenges. Information Technology and Libraries. (ITAL) 27(1):5-22.

Sero Consulting Ltd. 2008. An Evaluation and horizon scan of the current library management systems and related systems landscape for UK higher education. JISC \& SCONUL Library Management Systems Study, JISC \& SCONUL LMS. 157 p.

Tennant, Roy. 2005. Digital Libraries : «Lipstick on a Pig». Library Journal 7. Accédée le 28 août, 2008, http://www.libraryjournal. com/article/CA516027.html. 